\title{
Observational evidence for a connection between SMBHs and dark matter haloes
}

\section{Maarten Baes ${ }^{1,2}$, Herwig Dejonghe ${ }^{1}$, Pieter Buyle ${ }^{1}$, Laura Ferrarese ${ }^{3}$ and Gianfranco Gentile ${ }^{4,5}$}

\author{
${ }^{1}$ Sterrenkundig Observatorium, Universiteit Gent, \\ Krijgslaan 281 S9, B-9000 Gent, Belgium email: maarten.baes@ugent.be \\ ${ }^{2}$ Department of Physics and Astronomy, Cardiff University, \\ 5 The Parade, Cardiff CF243YB, Wales, UK \\ ${ }^{3}$ Rutgers University, New Brunswick, NJ 08854, USA \\ ${ }^{4}$ Radioastronomisches Institut der Universität Bonn, \\ Auf dem Hügel 71D, D-53121 Bonn, Germany \\ ${ }^{5}$ SISSA, via Beirut 4, 34014 Trieste, Italy
}

\begin{abstract}
We investigate the relation between circular velocity $v_{\mathrm{c}}$ and bulge velocity dispersion $\sigma$ in spiral galaxies, based on literature data and new spectroscopic observations. We find a strong, nearly linear $v_{\mathrm{c}}-\sigma$ correlation with a negligible intrinsic scatter, and a striking agreement with the corresponding relation for elliptical galaxies. The least massive galaxies $\left(\sigma<80 \mathrm{~km} \mathrm{~s}^{-1}\right)$ significantly deviate from this relation. We combine this $v_{\mathrm{c}}-\sigma$ correlation with the well-known $M_{\mathrm{BH}}-\sigma$ relation to obtain a tight correlation between circular velocity and supermassive black hole mass, and interpret this as observational evidence for a close link between supermassive black holes and the dark matter haloes in which they presumably formed. Apart from being an important ingredient for theoretical models of galaxy formation and evolution, the relation between $M_{\mathrm{BH}}$ and circular velocity has the potential to become an important practical tool in estimating supermassive black hole masses in spiral galaxies.
\end{abstract}

\section{Introduction}

The existence of supermassive black holes (SMBHs) in the nuclei of galaxies has been suspected for almost half a decade, as accretion onto SMBHs seemed the only logical explanation for the existence of quasars. HST observations have provided evidence that SMBHs with masses ranging from $10^{6}$ to $10^{9} \mathrm{M}_{\odot}$ are present in the centre of a few dozens of nearby (quiescent) galaxies. Be this sufficient evidence for the existence of SMBHs, we can now tackle more fundamental questions concerning their formation and evolution. An obvious way to proceed is the study of the relation between SMBHs and the galaxies that host them. It was found that black hole masses are correlated with parameters of the hot stellar components of their host galaxies. The tight $M_{\mathrm{BH}}-\sigma$ relation (Gebhardt et al. 2000; Ferrarese \& Merritt 2000) is now the preferred paradigm to study SMBH demographics in galactic nuclei.

This apparently tight link between bulges and SMBHs reflects an important ingredient that should be reproduced (and thus hopefully explained) by theoretical models of galaxy formation. In fact, the tightness of the $M_{\mathrm{BH}}-\sigma$ correlation is somewhat surprising. In most of the state-of-the-art models, the total galaxy mass (or dark matter mass $M_{\mathrm{DM}}$ ), rather than the bulge mass, plays a fundamental role in shaping the SMBHs. A close correlation could therefore be expected between $M_{\mathrm{BH}}$ and $M_{\mathrm{DM}}$, rather than between $M_{\mathrm{BH}}$ and the bulge properties. Establishing whether the $M_{\mathrm{BH}}-\sigma$ or the $M_{\mathrm{BH}}-M_{\mathrm{DM}}$ 
relation reflects the fundamental mode by which SMBHs form and evolve will ultimately rely on a comparison of the intrinsic scatter of the two correlations.

Unfortunately, a direct observational characterization of the $M_{\mathrm{BH}}-M_{\mathrm{DM}}$ relation is currently impossible. Ferrarese (2002b) first argued that a correlation between $M_{\mathrm{BH}}$ and $M_{\mathrm{DM}}$ should be reflected in an $M_{\mathrm{BH}}-v_{\mathrm{c}}$ correlation, where $v_{\mathrm{c}}$ is the circular velocity in the flat part of the rotation curve of spiral galaxies. Indeed, in most of the state-of-theart galaxy formation models, there is a one-to-one correspondence between the circular velocity and the mass of the dark matter halo. Unfortunately, there are (presently) only a handful of spiral galaxies with secure SMBH masses, and only two of them have a well-measured extended rotation curve. A way to avoid this problem is to adopt the tight $M_{\mathrm{BH}}-\sigma$ correlation in order to estimate black hole masses in a larger sample of galaxies. A tight correlation between SMBH mass and dark matter halo mass should thus appear in the form of a correlation between central velocity dispersion and circular velocity. Ferrarese (2002b) presented a first attempt at establishing such a correlation. Baes et al. (2003) significantly improved on these results by almost doubling the sample size. The present contribution is focused on the latter results.

\section{Sample selection}

A simple measure for the circular velocity of galaxies is half of the integrated line width from spatially unresolved HI $21 \mathrm{~cm}$ measurements, corrected for inclination. Various authors have recovered a nearly linear correlation between integrated line width and bulge velocity dispersion (e.g. Whitmore \& Kirshner 1981; Whittle 1992; Franx 1993). This correlation has significant scatter and galaxy type could act as a third parameter in this correlation. This can be due to the fact that the integrated line width is not an accurate measure for the flat part of the rotation curve, and hence of the total dark matter content. Therefore, we chose to consider only those galaxies with an extended rotation curve measured well beyond the optical radius, in order to reliably trace the circular velocity in the flat part of the rotation curve. In order to see the benefits of this approach, it is interesting to consider the Tully-Fisher (TF) study of the Ursa Major cluster spiral galaxies by Verheijen (2001): the scatter in the TF relation strongly decreases when he considers the flat part in the rotation curve instead of the integrated line width.

We constructed a data set of 28 spiral galaxies with central velocity dispersion data and a rotation curve measured beyond the optical radius. For 16 spirals, the data could be retrieved from the literature (see references in Ferrarese 2002b). For the remaining 12 galaxies, rotation curve data were available in the literature (Palunas \& Williams 2000), and the velocity dispersions were measured with the EFOSC2 instrument on the ESO $3.6 \mathrm{~m}$ telescope. The total sample of 28 spiral galaxies can be found in Baes et al. (2003).

\section{The $v_{\mathbf{c}}-\sigma$ correlation}

In the left panel of figure 1 we plot the circular velocity versus the velocity dispersion for the 28 spiral galaxies in our sample. For the 24 galaxies with a velocity dispersion greater than about $80 \mathrm{~km} \mathrm{~s}^{-1}$, there is a very tight correlation between $v_{\mathrm{c}}$ and $\sigma$. We fitted a straight line to these data, taking into account the errors on both quantities and obtained

$$
\log \left(\frac{v_{\mathrm{c}}}{v_{0}}\right)=(0.96 \pm 0.11) \log \left(\frac{\sigma}{v_{0}}\right)+(0.21 \pm 0.023)
$$



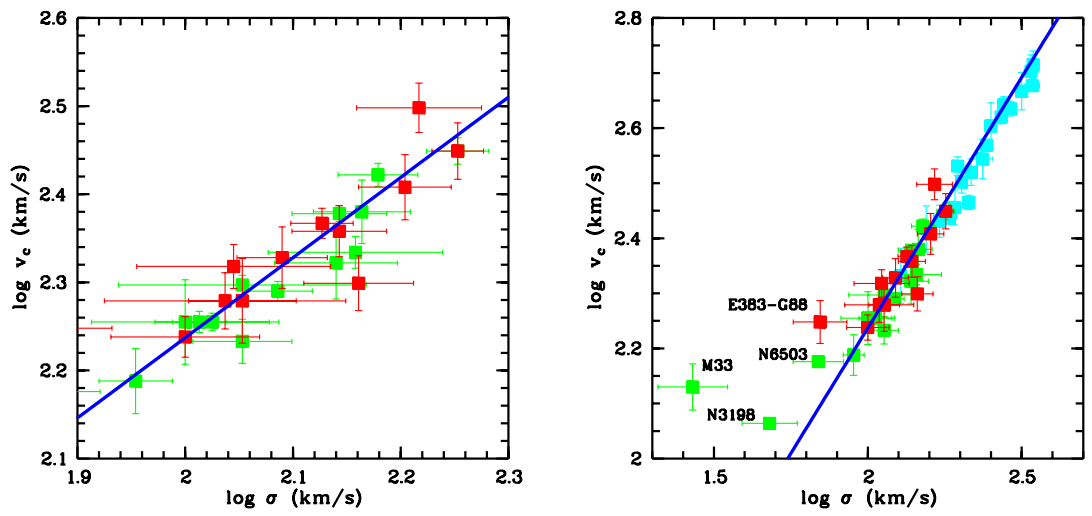

Figure 1. The correlation between the circular velocity $v_{\mathrm{c}}$ and the central velocity dispersion $\sigma$. The left plot shows the $v_{\mathrm{c}}-\sigma$ correlation for the 24 spiral galaxies with rotation curve beyond the optical radius and a velocity dispersion $\sigma>80 \mathrm{~km} \mathrm{~s}^{-1}$. The data points are from Ferrarese (2002b) and Baes et al. (2003) respectively. The right plot zooms out and adds the four spiral galaxies with $\sigma<80 \mathrm{~km} \mathrm{~s}^{-1}$ and the elliptical galaxies from Kronawitter et al. (2000).

where $v_{0}=200 \mathrm{~km} \mathrm{~s}^{-1}$. Two issues concerning this $v_{\mathrm{c}}-\sigma$ correlation deserve some special attention.

Firstly, the tightness of the correlation is astonishing: we find $\chi_{\text {red }}^{2}=0.281$, corresponding to a goodness-of-fit of 99.9 per cent. The $v_{\mathrm{c}}-\sigma$ relation can hence be regarded as having a negligible intrinsic scatter. Moreover, this correlation appears to be robust: there are no significant outliers in the range $\sigma>80 \mathrm{~km} \mathrm{~s}^{-1}$. The correlation appears to break down for galaxies with dispersions below about $80 \mathrm{~km} \mathrm{~s}^{-1}$ however: all four galaxies with $\sigma<80 \mathrm{~km} \mathrm{~s}^{-1}$ lie significantly above the correlation defined by the more massive spirals. Interestingly, this is also the mass range in which nearly all bulgeless spiral galaxies are located. New observations are indispensable to understand the behaviour of the $v_{\mathrm{c}}-\sigma$ correlation in the low mass regime. Moreover, all galaxies in our sample are high surface brightness galaxies, and it presently unclear how the $v_{\mathrm{c}}-\sigma$ relation behaves in diverse environments (see also Pizzella et al. 2004).

Secondly, it is interesting to compare this correlation to a similar one recently found for elliptical galaxies. Based on stellar dynamical models for 20 round ellipticals constructed by Kronawitter et al. (2000), Gerhard et al. (2001) discovered a very tight relation between the central dispersion and the circular velocity (the circular velocity curves of ellipticals were found to be flat to within 10 per cent). Both the slope and zero-point of this correlation agree amazingly well with the $v_{\mathrm{c}}-\sigma$ correlation of our spiral galaxy sample (see right panel of figure 1). Gerhard et al. (2001) argue that a proportionality between $\sigma$ and $v_{\text {c }}$ can be expected for ellipticals on the basis of their dynamical homology. For spiral galaxies this proportionality cannot be explained by simple dynamical arguments, as convincingly argued by Ferrarese (2002b). Moreover, the fact that both spiral and elliptical galaxies seem to obey exactly the same correlation is absolutely striking.

\section{The correlation between $M_{\mathrm{BH}}$ and $v_{\mathrm{c}}$}

As both the $v_{\mathrm{c}}-\sigma$ and $M_{\mathrm{BH}}-\sigma$ correlations seem to hold over the entire Hubble range, we can combine them to derive a correlation between the circular velocity and SMBH mass. Although the slope of the $M_{\mathrm{BH}}-\sigma$ relation is not well established (Tremaine et al. 2002; Ferrarese 2002a), this little affects the conclusion that the $v_{\mathrm{c}}-\sigma$ relation entails 
a connection between SMBH mass and the large scale velocity of the host galaxy (and henceforth the mass of the surrounding dark matter halo). For instance, using the characterization of the $M_{\mathrm{BH}}-\sigma$ relation from Tremaine et al. (2002), we obtain

$$
\log \left(\frac{M_{\mathrm{BH}}}{\mathrm{M}_{\odot}}\right)=(4.21 \pm 0.60) \log \left(\frac{v_{\mathrm{c}}}{v_{0}}\right)+(7.24 \pm 0.17) .
$$

The correlation between SMBH mass and circular velocity is useful for two different goals. Firstly, combined with other tight relations such as the $M_{\mathrm{BH}}-\sigma$ relation and the $\mathrm{TF}$ relation, it clearly points at an intimate interplay between the various galactic components (dark matter, discs, bulges and SMBHs) and forms a strong test for galaxy formation and evolution models. In particular, the $v_{\mathrm{c}}-\sigma$ relation can be used to discriminate between various theoretical models of galaxy formation. For example, if SMBHs form mainly through coalescence of smaller black holes during galaxy mergers, a relation $M_{\mathrm{BH}} \propto v_{\mathrm{c}}^{3}$ is expected, whereas theories in which accretion and feedback are the main ingredients for black hole growth prefer a $M_{\mathrm{BH}} \propto v_{\mathrm{c}}^{5}$ relation (e.g. Silk \& Rees 1998; Haiman \& Loeb 1998; Haehnelt, Natarajan \& Rees 1998; Kauffman \& Haehnelt 2000; Wyithe \& Loeb 2003; Di Matteo et al. 2003).

Apart from being an ingredient in theoretical galaxy formation models, the derived $M_{\mathrm{BH}}-v_{\mathrm{c}}$ relation can also serve as a practical tool to estimate the black hole masses in spiral galaxies. The most preferred means of estimating $M_{\mathrm{BH}}$ in galaxies is the $M_{\mathrm{BH}}-\sigma$ relation. Unfortunately, the number of spiral galaxies with reliable velocity dispersion measurements is relatively small. Since extended rotation curves have been measured for large samples of spiral galaxies (mainly for use in $\mathrm{TF}$ studies), the $M_{\mathrm{BH}}-v_{\mathrm{c}}$ relation has the potential to become an important practical tool in estimating supermassive black hole masses in spiral galaxies.

\section{References}

Baes, M., Buyle, P., Hau, G. K. T., \& Dejonghe, H. 2003, MNRAS, 341, L44

Di Matteo, T., Croft, R. A. C., Springel, V., \& Hernquist, L. 2003, ApJ, 593, 56

Ferrarese, L. 2002a, in Current high-energy emission around black holes, (eds. C. Lee and H. Chang), p. 3

Ferrarese, L. 2002b, ApJ, 578, 90

Ferrarese, L., \& Merritt, D. 2000, ApJ, 539, L9

Franx, M. 1993, IAUS, 153, 243

Gebhardt, K., et al. 2000, ApJ, 539, L13

Gerhard, O., Kronawitter, A., Saglia, R. P., \& Bender, R. 2001, AJ, 121, 1936

Haehnelt, M. G., Natarajan, P., \& Rees, M. J. 1998, MNRAS, 300, 817

Haiman, Z., \& Loeb, A. 1998, ApJ, 503, 505

Kauffmann, G., \& Haehnelt, M. 2000, MNRAS, 311, 576

Kormendy, J., \& Richstone, D. 1995, ARA\&A, 33, 581

Kronawitter, A., Saglia, R. P., Gerhard, O., \& Bender, R. 2000, A\&AS, 144, 53

Palunas, P., \& Williams, T. B. 2000, AJ, 120, 2884

Pizzella, A., Dalla Bontà, E., Corsini, E. M., Coccato, L., Sarzi, M., \& Bertola, F. 2004, this volume

Silk, J., \& Rees, M. J. 1998, A\&A, 331, L1

Tremaine, S., et al. 2002, ApJ, 574, 740

Verheijen, M. A. W. 2001, ApJ, 563, 694

Whitmore, B. C., \& Kirshner, R. P. 1981, ApJ, 250, 43

Whittle, M. 1992, ApJ, 387, 109

Wyithe, J. S. B., \& Loeb, A. 2003, ApJ, 595, 614 